\title{
Mating Stimulation Required for Mating-Induced Estrous Abbreviation in Female Rats: Effects of Repeated Testing
}

\author{
Amy L. Bennett,*† Meg E. Blasberg,* and J effrey D. Blaustein*,1 \\ ${ }^{*}$ Center for Neuroendocrine Studies, Neuroscience and Behavior Program, University \\ of Massachusetts, Amherst, Massachusetts 01003; and +Psychology Department, \\ Division of Social Sciences, Concordia University, Ann Arbor, Michigan 48105
}

Received October 22, 2001; revised January 28, 2002; accepted January 29, 2002

Mating stimulation, particularly vaginal- cervical stimulation, causes estrous abbreviation in female rats. In most previous studies, female rats were repeatedly tested for sexual behavior until estrous termination occurred. Thus, it was not clear whether sensory stimulation (e.g., flank stimulation, olfactory cues) received during the repeated testing procedure contributed to estrous abbreviation. In Experiment 1, we determined the effect of premating to two or four ejaculations on the rate of estrous termination when a repeated testing procedure was used. We compared ovariectomized, hormone-primed, female rats receiving (1) four ejaculations, (2) two ejaculations, or (3) no premating. Females premated to either two or four ejaculations showed significantly lower levels of sexual receptivity $12 \mathrm{~h}$ later than did nonpremated females. These results confirm that premating induces estrous abbreviation when a repeated testing procedure is used. In Experiment 2, we determined whether the repeated testing procedure was necessary for estrous abbreviation. Ovariectomized, hormone-primed female rats were premated to two ejaculations or not premated. The rats were then tested for sexual behavior repeatedly or only once. Females that were premated and repeatedly tested for sexual behavior showed a statistically significant decrease in sexual receptivity compared to females that were not premated; however, the level of sexual receptivity in premated females did not differ from that in non-premated females when they were tested only once. The results suggest that heat duration is the result of a complex interplay between those factors that promote the expression of sexual receptivity and those that inhibit it. (c) 2002 Elsevier Science (USA)

${ }^{1}$ To whom correspondence should be addressed at, Center for Neuroendocrine Studies, Neuroscience and Behavior Program, Tobin Hall, 135 Hicks Way, University of Massachusetts, Amherst, MA 01003-9271. Fax: (413) 545-0769. E-mail: blaustein@cns.umass.edu.
In estrous cycling female rats, a gradual increase in estradiol levels followed by a peak in progesterone induces sexual behavior (Boling and Blandau, 1939; Powers, 1970). In one early study, the period of sexual receptivity lasted an average of $13.7 \mathrm{~h}$ (Blandau, Boling, and Young, 1941). Although ovariectomy abolishes spontaneous estrus by removing the main source of these hormones, sexual behavior can be reinstated by sequential injections of estradiol and progesterone (Boling and Blandau, 1939).

Many aspects of female sexual behavior are influenced not only by hormones, but also by the sensory stimulation that the female receives during mating. Although the female receives many types of sensory cues (e.g., olfactory, auditory, and tactile) during mating, previous studies suggest that vaginal-cervical stimulation (VCS) received during intromissions and ejaculations is particularly important for inducing changes in sexual behavior and reproductive physiology. For example, VCS increases return latencies during paced mating (Yang and Clemens, 1997) and induces twice daily prolactin surges necessary to maintain pregnancy (Everett, 1964).

The duration of sexual receptivity is also influenced by mating stimulation. In an earlier study, mating stimulation reduced estrous duration by about $3 \mathrm{~h}$ (Blandau et al., 1941). Like changes in return latencies and the induction of pseudopregnancy, estrous abbreviation is believed to be induced specifically by VCS. Either transection of the pelvic nerve, a primary source of sensory afferents from the cervix (Lodder and Zeilmaker, 1976), or covering the vagina, which blocks the receipt of intromissions (Reading and Blaustein, 1984), prevents mating-induced estrous abbreviation. Furthermore, females receiving experimenter-administered VCS show behavioral changes 
similar to those observed following mating, such as increased rejection behaviors and decreased lordosis magnitude (Pfaus, Smith, Byrne, and Stephens, 2000). The temporal pattern of VCS is also important. Intromissions received when the female is allowed to pace the mating sequence are more effective in inducing estrous abbreviation than intromissions received when the male paces mating (Coopersmith, Candurra, and Erskine, 1996; Erskine, 1985).

Unfortunately, the amounts of mating stimulation used in previous studies have been variable. For example, in some estrous abbreviation studies, females have been mated with two males for $2 \mathrm{~h}$ (Reading and Blaustein, 1984), while other have used smaller amounts of stimulation, such as mating females only until 10 paced intromissions were received (Erskine, 1985). Furthermore, in most previous studies changes in sexual behavior were measured using a withinsubjects testing technique in which rats were vaginally masked and repeatedly tested every 1 or $2 \mathrm{~h}$ until the termination of sexual behavior (Blandau et al., 1941; Coopersmith et al., 1996; Erskine, 1985; Lodder and Zeilmaker, 1976; Reading and Blaustein, 1984). It is not clear whether sensory stimulation (e.g., flank stimulation or olfactory cues) received during repeated testing also contributes to the rate of estrous abbreviation.

Several studies suggest that repeated mating can alter the expression of female sexual behavior (Auger, Moffatt, and Blaustein, 1997; Bennett, Blasberg, and Blaustein, 2001; Dudley and Moss, 1994; Foreman and Moss, 1977; Hardy and DeBold, 1973; Rajendren, Dudley, and Moss, 1990; Rajendren, Dudley, and Moss, 1991; Rajendren and Moss, 1993, 1994). Although VCS seems to be important for these alterations (Bennett $e t$ al., 2001), other sensory cues such as odors (Rajendren et al., 1991; Rajendren and Moss, 1994) and other types of tactile stimulation (Hardy and DeBold, 1973) also contribute to the effects of repeated mating on subsequent behavior. Therefore, although VCS is typically prevented during repeated testing for estrous abbreviation, it is possible that other sensory cues received during repeated testing contribute to changes in sexual behavior.

In order to determine the contribution of repeated testing, we first determined an appropriate amount of mating stimulation to use in the study. In Experiment 1 , we determined the effects of three different levels of premating stimulation (four ejaculations, two ejaculations, or no mating) on the rate of estrous termination when females subsequently were repeatedly tested for sexual receptivity. Then in Experiment 2, we tested the hypothesis that the repeated testing procedure itself contributes to mating-induced estrous abbreviation by comparing females receiving either premating to two ejaculations or no premating that were then either repeatedly tested for sexual receptivity or tested only once.

\section{METHOD}

\section{General}

Female Sprague-Dawley rats (175-200 g) obtained from Charles River Breeding Laboratories, Inc. (Wilmington, MA) were group housed in a 14:10 h light: dark cycle. Purina LabDiet 5001 and water were available ad libitum throughout the experiment. At least 1 week after arrival, the rats were bilaterally ovariectomized under $2.5 \mathrm{mg} / \mathrm{kg}$ acepromazine, $10 \mathrm{mg} / \mathrm{kg}$ xylazine, and $50 \mathrm{mg} / \mathrm{kg}$ ketamine. All procedures were approved by the Institutional Animal Care and Use Committee at the University of Massachusetts, Amherst.

One week after surgery, the rats were injected subcutaneously with $2 \mu \mathrm{g}$ estradiol benzoate (EB; dissolved in $0.1 \mathrm{ml}$ sesame oil). Forty-four hours later, they were injected subcutaneously with $500 \mu \mathrm{g}$ progesterone (dissolved in $0.1 \mathrm{ml}$ sesame oil containing $5 \%$ benzyl alcohol and $15 \%$ benzyl benzoate). Four hours later, the rats were pretested for sexual receptivity, and lordosis quotients (number of lordosis responses divided by the number of mounts by the male, multiplied by 100) were recorded. Each female was left with a male until 10 mounts were received. All behavior tests took place during the dark phase of the light:dark cycle in cubic Plexiglas arenas (33.7 $\mathrm{cm} \times 37.0 \mathrm{~cm} \times 32.0 \mathrm{~cm}$ ) lined with wood chips. Two females displayed low levels of sexual receptivity (lordosis quotient $\leq 70$ ) at the time of the pretest and were not included in the studies.

\section{Experiment 1}

Ten days after the pretest, rats were injected with 2 $\mu \mathrm{g}$ EB followed $44 \mathrm{~h}$ later by $500 \mu \mathrm{g}$ progesterone. Four hours after the progesterone injection, the rats received one of three treatments: (1) premated with a male rat until two ejaculations were received and then premated with another male until two additional ejaculations were received $(n=6) ;(2)$ premated with a male rat until two ejaculations were received $(n=6)$; or (3) placed in an empty arena until the end of the premating treatment $(n=6)$. Each male was only used once during the experiment. Beginning $4 \mathrm{~h}$ after the start of the treatment the females were tested for 
sexual receptivity every $2 \mathrm{~h}$ for the next $8 \mathrm{~h}(4,6,8,10$, and $12 \mathrm{~h}$ after treatment). All rats were maintained in a room illuminated by red light for the duration of testing. During testing, each rat was vaginally masked (a small piece of duct tape was placed over the vaginal and perineal region and secured to the base of the tail) to prevent receipt of intromissions and placed with a male rat until 10 mounts were received. Lordosis quotients were recorded by an observer blind to the treatment condition. Data were analyzed with a 3 (treatment) $\times 5$ (time) factor repeated-measures ANOVA. Significant results were analyzed with NewmanKeuls post hoc comparisons. Results were considered statistically significant if $P<0.05$.

\section{Experiment 2}

Ten days after the pretest, rats were injected with 2 $\mu \mathrm{g}$ EB followed $44 \mathrm{~h}$ later by $500 \mu \mathrm{g}$ progesterone. Four hours after the progesterone injection, the females were either (1) premated with a male rat until two ejaculations were received or (2) vaginally masked and placed with a male rat until three mounts were received (to verify sexual receptivity) and then placed in an arena with another female until the end of the premating treatment. Each male was only used once during the experiment. Beginning $6 \mathrm{~h}$ after the start of the treatment, all rats were vaginally masked and tested for sexual receptivity with a male rat until 10 mounts were received. Females were tested under one of four conditions: (1) repeatedly tested at 6, 10, and $14 \mathrm{~h}$ after the start of treatment; (2) tested once $6 \mathrm{~h}$ after treatment; (3) tested once $10 \mathrm{~h}$ after treatment; or (4) tested once $14 \mathrm{~h}$ after treatment. There were a total of eight groups (7-8 rats/group). Test times were chosen based on the results of Experiment 1 to include the following: (1) a time point when all females were expected to show high levels of sexual behavior $(6 \mathrm{~h})$; (2) a time point when only premated females were expected to show low levels of sexual behavior $(10 \mathrm{~h})$; and (3) a time point when all females were expected to show low levels of sexual behavior $(14 \mathrm{~h})$. All rats were maintained in a room illuminated by red light for the duration of testing. Lordosis quotients were recorded by an observer blind to the treatment condition. Data from females tested repeatedly at 6,10 , and $14 \mathrm{~h}$ after treatment were analyzed using a 2 (treatment) $\times 3$ (time) repeated-measures ANOVA. Statistically significant effects were analyzed with Newman-Keuls post hoc comparisons. Data from females tested once at 6,10 , or $14 \mathrm{~h}$ were analyzed using a 2 (treatment) $\times 3$ (time) factor ANOVA. Significant effects were analyzed with Newman-Keuls post hoc

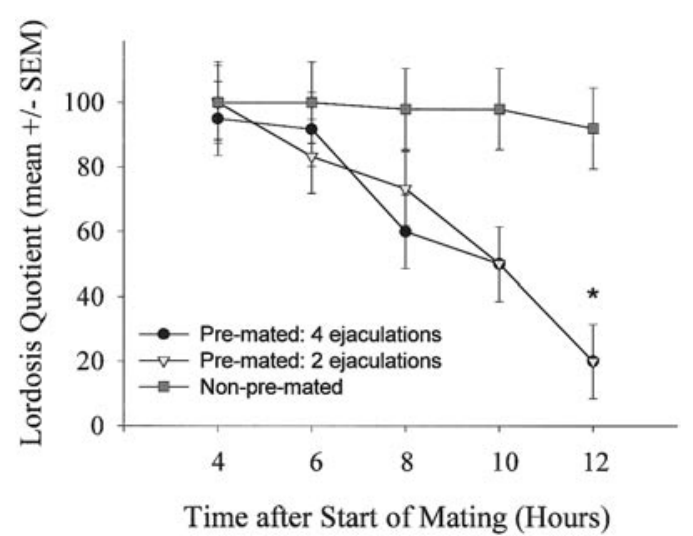

FIG. 1. Lordosis quotients of premated females receiving four ejaculations $(n=6)$, premated females receiving two ejaculations $(n=6)$, and non-premated females $(n=6) 4,6,8,10$, and $12 \mathrm{~h}$ after treatment.

comparisons. Results were considered statistically significant if $P<0.05$.

\section{RESULTS}

\section{Experiment 1}

Premating to either two or four ejaculations caused a significant decrease in lordosis quotient $10 \mathrm{~h}$ later (Fig. 1). A $3 \times 5$ repeated-measures ANOVA revealed a significant main effect of treatment $(F=4.38, P<$ $0.05)$ and of time $(F=18.06, P<0.001)$, as well as a significant interaction $(F=3.35, P<0.01)$. Premated females receiving either two or four ejaculations had significantly lower lordosis quotients at the 12-h test $(P<0.05)$ than did non-premated females. In addition, premated females receiving either two or four ejaculations had significantly lower lordosis quotients at the $10-(P<0.05)$ and $12-(P<0.05) \mathrm{h}$ tests than at the 4 - and 6-h tests. Females that were not premated did not show a change in lordosis quotient during the five tests. There were no statistically significant differences in lordosis quotient between females premated to two or four ejaculations at any time point.

\section{Experiment 2}

Repeatedly tested females. As in Experiment 1, females premated to two ejaculations showed a more rapid termination of sexual behavior than did nonpremated females. Although there was not a significant main effect of treatment, a $2 \times 3$ repeated-measures ANOVA revealed a significant main effect of time $(F=28.4, P<0.001)$ and a significant interac- 


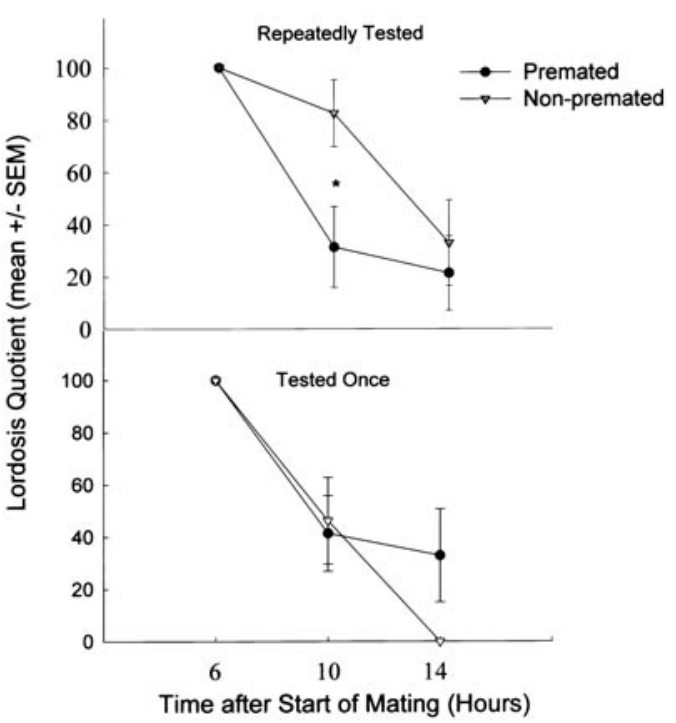

FIG. 2. Lordosis quotients of premated and non-premated female rats 6,10 , and $14 \mathrm{~h}$ after treatment (top). Lordosis quotients of premated and non-premated females tested once at 6,10 , or $14 \mathrm{~h}$ after treatment (bottom). $n=7-8$ rats per group.

tion $(F=3.97, P<0.05)$ in females that were repeatedly tested (Fig. 2, top). Post hoc comparisons revealed that premated females had significantly lower lordosis quotients at the 10-h test than did non-premated females $(P<0.05)$. In addition, premated females had significantly lower lordosis quotients at the 10 -h $(P<$ $0.05)$ and 14 -h $(P<0.05)$ tests than at the 6-h test. Non-premated females had significantly lower lordosis quotients at the 14 -h test than at the 6-h $(P<0.05)$ and 10-h $(P<0.05)$ tests.

Females tested once. Although all groups showed a decrease in sexual behavior over time, there was no effect of premating on the rate of estrous termination. A $2 \times 3$ factor ANOVA revealed a significant main effect of time $(F=28.56, P<0.001)$ in females retested once at 6,10 , or $14 \mathrm{~h}$; however, there was not a significant effect of treatment or a significant interaction (Fig. 2, bottom). Both premated and non-premated females showed significantly lower lordosis quotients at the 10-h $(P<0.05)$ and 14-h $(P<0.05)$ tests than at the 6-h test.

\section{DISCUSSION}

In Experiment 1, females premated to two or four ejaculations showed a significant decrease in sexual receptivity at the 12-h test compared to non-premated females. This finding is consistent with previous studies suggesting that mating induces estrous abbrevia- tion in female rats (Blandau et al., 1941; Coopersmith et al., 1996; Erskine, 1985; Lodder and Zeilmaker, 1976; Reading and Blaustein, 1984). These findings suggest that two ejaculations are sufficient to induce estrous abbreviation when females are tested repeatedly for estrous termination. Furthermore, there was no difference in the rate of estrous abbreviation following two or four ejaculations, suggesting that four ejaculations do not induce estrous abbreviation more effectively than two ejaculations. However, we cannot rule out the possibility that more than four ejaculations may induce estrous abbreviation at a more rapid rate.

In Experiment 2, when females were repeatedly tested for sexual behavior, females premated to two ejaculations showed significantly lower levels of sexual receptivity at the 10 -h test than did non-premated females. These findings are consistent with previous studies that demonstrated mating-induced estrous abbreviation using a repeated testing technique (Blandau et al., 1941; Coopersmith et al., 1996; Erskine, 1985; Lodder and Zeilmaker, 1976; Reading and Blaustein, 1984). However, females premated to two ejaculations did not differ from non-premated females when the rats were tested only once. These results suggest that sensory stimulation (e.g., olfactory cues, flank stimulation) received during repeated testing affects the rate of estrous termination.

Although a previous study found that mating-induced estrous abbreviation occurred in the absence of repeated testing (Reading and Blaustein, 1984), methodological differences between the previous and current studies may account for the differing results. In the previous study, female rats were premated with two males for $2 \mathrm{~h}$. It is possible that this greater amount of mating stimulation is sufficient to induce estrous abbreviation in the absence of repeated testing, while the two ejaculations used in the current study were not. Although it was not tested directly in this earlier experiment, it is possible that mating with two males for $2 \mathrm{~h}$ in a small enclosure results in vaginal trauma, which can cause decreases in sexual receptivity (van der Schoot, van Ophemert, and Baumgarten, 1992).

VCS is necessary for mating to induce estrous abbreviation (Lodder and Zeilmaker, 1976; Reading and Blaustein, 1984). Furthermore, experimenter-administered VCS in the absence of other sensory cues increases rejection behaviors and decreases lordosis magnitude (Pfaus et al., 2000). However, experimenteradministered VCS does not result in the significant decreases in lordosis quotient usually observed following mating with a male rat (Pfaus et al., 2000), suggesting that other sensory cues also contribute to 
mating-induced estrous abbreviation. Because the females were masked during testing, the current results also suggest that types of sensory stimulation, other than VCS, contribute to changes in sexual receptivity. It is possible that VCS works in combination with other sensory cues (e.g., olfactory cues and flank stimulation) to induce these changes.

However, it is important to note that, among the non-premated rats tested at $10 \mathrm{~h}$, those that were repeatedly tested appeared to maintain a higher level of sexual receptivity than did those tested once. These findings suggest that sensory stimulation received during repeated testing does not actually contribute to the termination of sexual behavior in premated females, but rather results in maintenance of a high level of sexual behavior in non-premated females. It is not surprising that repeated testing increases the level of sexual behavior in non-premated females. A considerable body of research suggests that repeated mating of female rats enhances sexual receptivity in females that have not been premated (Auger et al., 1997; Bennett et al., 2001; Dudley and Moss, 1994; Foreman and Moss, 1977; Hardy and DeBold, 1973; Rajendren et al., 1990; 1991; Rajendren and Moss, 1993, 1994). Although these increases in sexual behavior have been attributed mainly to VCS received during mating (Bennett et al., 2001), it is important to note that under some experimental conditions repeated mating in the absence of intromissions is sufficient to increase sexual behavior (Hardy and DeBold, 1973). Some evidence suggests that these increases may be mediated by olfactory cues received during mating (Rajendren et al., 1991; Rajendren and Moss, 1994). Other sensory cues (e.g., flank stimulation) may also be involved in increases in sexual behavior following repeated mating; however, their effects have not been directly tested. Therefore, odors as well as other sensory cues (e.g., flank stimulation) received during repeated testing could maintain a high level of sexual behavior in the non-premated females. The precise sensory cues responsible for the enhancement are under investigation.

The mechanism by which mating stimulation causes decreases in sexual behavior is not yet known. Activation of progestin receptors by progesterone causes down-regulation of the receptors, which induces hyposensitivity to progesterone, resulting in a loss of the behavioral response (Blaustein and Olster, 1989). Because mating stimulation, particularly VCS, is believed to cause ligand-independent activation of progestin receptors (Auger et al., 1997), it is possible that mating stimulation also induces down-regulation of progestin receptors leading to the hastened termination of sexual receptivity. Alternatively, it has been suggested that activation of estrogen receptor- $\beta$ $(\mathrm{ER} \beta)$-containing cells may contribute to the termination of sexual behavior (Greco, Blasberg, Kosinski, and Blaustein, unpublished results). Although ER $\beta$ genedisrupted mice show normal sexual behavior, they continue to show sexual behavior considerably longer than do wild-type controls (Ogawa, Chan, Chester, Gustafsson, Korach, and Pfaff, 1999), suggesting that $\mathrm{ER} \beta$ is necessary for the normal termination of sexual behavior. Thus, ER $\beta$ may contribute to the termination and/or abbreviation of sexual receptivity.

Regardless of the mechanism by which mating abbreviates sexual receptivity, the current results suggest that the effects of mating on subsequent behavior are more complex than previously appreciated. For example, in some cases, mating stimulation, such as VCS (Bennett et al., 2001) and odor (Rajendren et al., 1990; Rajendren and Moss, 1994), contributes to increases in sexual behavior. However, under some conditions, VCS contributes to subsequent decreases in sexual receptivity approximately $10 \mathrm{~h}$ later (Lodder and Zeilmaker, 1976; Reading and Blaustein, 1984). These interactions may serve to ensure that under the appropriate hormonal conditions female rats will become and remain receptive in the presence of males until they receive sufficient mating stimulation to result in pregnancy. Once sufficient stimulation is received, sexual receptivity may terminate, allowing the female to minimize energy expenditure and the risk of predation resulting from mating.

In summary, Experiment 1 showed that premating to two or four ejaculations was sufficient to induce estrous abbreviation in female rats when a repeated testing procedure was used. In Experiment 2, females that were premated to two ejaculations showed more rapid estrous termination than did non-premated females when the repeated testing procedure was used, but not when the females were tested only once. Interestingly, estrous abbreviation seems to be apparent when a repeated testing procedure is used, because sensory cues received during repeated testing maintain higher levels of sexual behavior in non-premated females. Thus, heat duration seems to be a result of complex interactions between factors that promote sexual receptivity and those that inhibit it.

\section{ACKNOWLEDGMENTS}

This work was supported by NS19327 (J.D.B.), Senior Scientist Award MH01312 (J.D.B.), NRSA fellowship MH12474 (A.L.B.), and NRSA fellowship HD40410 (M.E.B.) from the National Institutes of Health. We thank Beth Lux for her expert technical assistance. 


\section{REFERENCES}

Auger, A. P., Moffatt, C. A., and Blaustein, J. D. (1997). Progesterone-independent activation of rat brain progestin receptors by reproductive stimuli. Endocrinology 138, 511-514.

Bennett, A. L., Blasberg, M. E., and Blaustein, J. D. (2001). Sensory cues mediating mating-induced potentiation of sexual receptivity in female rats. Horm. Behav. 40, 77-83.

Blandau, R. J., Boling, J. L., and Young, W. C. (1941). The length of heat in the albino rat as determined by the copulatory response. Anat. Rec. 79, 453-463.

Blaustein, J. D., and Olster, D. H. (1989). Gonadal steroid hormone receptors and social behavior. In J. Balthazart (Ed.), Advances in Comparative and Environmental Physiology, Vol. 3, pp. 31-104. Springer-Verlag, Berlin/Heidelberg.

Boling, J. L., and Blandau, R. J. (1939). The estrogen-progesterone induction of mating responses in the spayed female rat. Endocrinology 25, 359-364.

Coopersmith, C., Candurra, C., and Erskine, M. S. (1996). Effects of paced mating and intromissive stimulation on feminine sexual behavior and estrus termination in the cycling rat. J. Comp. Physiol. 110, 176-186.

Dudley, C. A., and Moss, R. L. (1994). Lesions of the accessory olfactory bulb decrease lordotic responsiveness and reduce mating-induced $c$-fos expression in the accessory olfactory system. Brain Res. 642, 29-37.

Erskine, M. S. (1985). Effects of paced coital stimulation on estrus duration in intact cycling rats and ovariectomized and ovariectomized-adrenalectomized hormone-primed rats. Behav. Neurosci. 99, 151-161.

Everett, J. W. (1964). Central neural control of reproductive function of the adenohypophysis. Physiol. Rev. 44, 373.

Foreman, M. M., and Moss, R. L. (1977). Effects of subcutaneous injection and intrahypothalamic infusion of releasing hormones upon lordotic response to repetitive coital stimulation. Horm. Behav. 8, 219-234.

Hardy, D. F., and DeBold, J. F. (1973). Effects of repeated testing on sexual behavior of the female rat. J. Comp. Physiol. Psych. 85, 195-202.

Lodder, J., and Zeilmaker, G. H. (1976). Role of the pelvic nerves in the post-copulatory abbreviation of behavioral estrus in female rats. J. Comp. Physiol. Psych. 90, 925-929.

Ogawa, S., Chan, J., Chester, A. E., Gustafsson, J. A., Korach, K. S., and Pfaff, D. W. (1999). Survival of reproductive behaviors in estrogen receptor $\beta$ gene-deficient $(\beta E R K O)$ male and female mice. Proc. Natl. Acad. Sci. USA 96, 12887-12892.

Pfaus, J. G., Smith, W. J., Byrne, N., and Stephens, G. (2000). Appetitive and consummatory sexual behaviors of female rats in bilevel chambers. II. Patterns of estrus termination following vaginocervical stimulation. Horm. Behav. 37, 96-107.

Powers, J. B. (1970). Hormonal control of sexual receptivity during the estrous cycle of the rat. Physiol. Behav. 5, 831-835.

Rajendren, G., Dudley, C. A., and Moss, R. L. (1990). Role of the vomeronasal organ in the male-induced enhancement of sexual receptivity in female rats. Neuroendocrinology 52, 368-372.

Rajendren, G., Dudley, C. A., and Moss, R. L. (1991). Role of the ventromedial nucleus of hypothalamus in the male-induced enhancement of lordosis in female rats. Physiol. Behav. 50, 705-710.

Rajendren, G., and Moss, R. L. (1993). The role of the medial nucleus of amygdala in the mating-induced enhancement of lordosis in female rats: The interaction with luteinizing hormone-releasing hormone neuronal system. Brain Res. 617, 81-86.

Rajendren, G., and Moss, R. L. (1994). Vomeronasal organ-mediated induction of fos in the central accessory olfactory pathways in repetitively mated female rats. Brain Res. 34, 53-59.

Reading, D. S., and Blaustein, J. D. (1984). The relationship between heat abbreviation and neural progestin receptors in female rats. Physiol. Behav. 32, 973-981.

van der Schoot, P., van Ophemert, J., and Baumgarten, R. (1992). Copulatory stimuli in rats induce heat abbreviation through effects on genitalia but not through effects on central nervous mechanisms supporting the steroid hormone-induced sexual responsiveness. Behav. Brain Res. 49, 213-223.

Yang, L. Y., and Clemens, L. G. (1997). Function of intromissions on intromission-return latency of female rats during paced sexual behavior. Physiol. Behav. 61, 889-894. 